\title{
Mean-variance hedging strategies in discrete time and continuous state space
}

\author{
O. L. V. Costa ${ }^{1}$, A. C. Maiali ${ }^{1}$ \& A. de C. Pinto ${ }^{2}$ \\ ${ }^{1}$ Escola Politécnica - Universidade de São Paulo, Brazil \\ ${ }^{2}$ Fundação Getulio Vargas - EAESP, Brazil
}

\begin{abstract}
In this paper we consider the mean-variance hedging problem of a continuous state space financial model with the rebalancing strategies for the hedging portfolio taken at discrete times. An expression is derived for the optimal self-financing mean-variance hedging strategy problem, considering any given payoff in an incomplete market environment. To some extent, the paper extends the work of Černý [1] to the case in which prices may assume any value within a continuous state space, a situation that more closely reflects real market conditions. An expression for the "fair hedging price" for a derivative with any given payoff is derived. Closed-form solutions for both the "fair hedging price" and the optimal control for the case of a European call option are obtained. Numerical results indicate that the proposed method is consistently better than the Black and Scholes approach, often adopted by practitioners.

Keywords: discrete-time mean-variance hedging, options pricing, optimal control.
\end{abstract}

\section{Introduction}

The problem of hedging options has systematically been the focus of attention from both researchers and practitioners alike. The complex nature of most derivatives has led academics to often simplify the conditions under which trading occurs, proposing models which, albeit computational and mathematically treatable, do not capture all of the peculiarities of these instruments. When modelling the dynamics of an asset price, its derivatives and the corresponding hedging process, the choices of state space and time parameter are determined so as to simplify the model's complexity. However, with respect to hedging, the situation that more closely follows what is observed in real market conditions is the use 
of discrete times for representing portfolio rebalancing instants, and continuous state spaces for values possibly assumed by prices. Indeed, decisions regarding rebalancing the hedged position naturally occur at discrete times, whereas the smallest possible price variation ("market ticks") can be more adequately modelled within a continuous state space framework. It is, therefore, the purpose of this work to solve, for a given option, the mean-variance hedging problem of a continuous state space financial model with the rebalancing strategies for the hedging portfolio taken at discrete times.

Most studies of mean-variance hedging to date have considered the case of rebalancing strategies taken at continuous time. For discrete-time rebalancing, various intertemporal mean-variance criteria were analysed by Schäl [2] in the case of a constant investment opportunity set. A solution for the general problem with one asset and non-stochastic interest rate, which does not have a fully recursive structure, was presented by Schweizer [3]. This difficulty was overcome by the work of Bertsimas [4], who presented a fully recursive dynamic programming solution for the case of one basis asset and non-stochastic interest rate. Černý [1] proposed a general and simple recursive solution for the hedging problem with stochastic interest rate and an arbitrary number of basis assets.

The purpose of this work is to extend the work of Černý [1] to the case where the dynamics of a risky asset price is represented by an Itô diffusion with constant parameters. This approach allows us to obtain expressions for both the fair hedging price (mean-value process) of the option to be hedged, and the optimal control to be applied at any rebalancing instant. In particular, we derive closed-form solutions for the case of European vanilla call options which eliminate the recursiveness of previous models, thus producing considerable computational gains.

The paper is organized as follows. Section 2 presents the basic model and the proposed method which produces non-recursive expressions for the mean value process of an option with any given payoff and its corresponding optimal control at any rebalancing instant. Section 3 applies the methodology described in Section 2 to the case of a European vanilla call option deriving closed-form expressions for the option value and for the amount of underlying asset to be bought or sold for hedging purposes, i.e. the optimal control. Numerical results comparing hedging strategies suggested by the optimal self-financing mean-variance hedging proposed in this paper and that by the Black and Scholes (B\&S) [5] approach are presented in Section 4. Finally, a summary and brief conclusions are presented in Section 5 .

\section{Discrete time, continuous state space mean-variance hedging strategy}

Let $t \in\left[0, T^{c}\right]$ represent a particular time instant in a continuous-time model, and $\tau \in\{0,1, \cdots, T\}$ represent the corresponding time instant in a discretetime model. Consider that the time interval between two consecutive discretetime instants is $\Delta t$, and that, for a particular $\tau$ whose corresponding continuous- 
time instant is $t$, we have that $T-\tau=n$, with $n$ being given by $n=\left(T^{c}-\right.$ $t) / \Delta t$.

Let $S(t)$ denote the price of a dividend-paying asset at time $t$. We assume that $S(t)$ follows a geometric Brownian motion described, in the continuous time setting notation, by the stochastic process below:

$$
S(t+\Delta t)=S(t) e^{\sigma \Delta W^{P}(t+\Delta t)+\left(\mu-\rho-\frac{1}{2} \sigma^{2}\right) \Delta t},
$$

and in the discrete-time setting notation, by:

$$
S(\tau+1)=S(\tau) e^{\sigma \Delta W^{P}(\tau+1)+\left(\mu-\rho-\frac{1}{2} \sigma^{2}\right) \Delta t} .
$$

The parameter $\mu$ represents the asset's expected rate of return; $\rho$, the asset's dividend yield; and $\sigma$, the volatility, all assumed to be constant. $W^{P}(\cdot)$ is a Wiener process under the probability measure $P$.

In a discrete-time setting, consider a market free of arbitrage opportunities composed of a risky asset $S$ and a risk-free asset $S^{0}$, whose value at discrete time $\tau$ is $S^{0}(\tau)$. The risk-free interest rate, $r$, is assumed to be constant, for all $\tau \in\{0,1, \cdots, T\}$, with $S^{0}$ and $r$ being related by $S^{0}(\tau+1)=S^{0}(\tau) e^{r \Delta t}$, with $S^{0}(0)=1$.

Let $H$ be a non-attainable derivative, maturing at time $\tau=T$, whose underlying asset is $S$. The derivative payoff is $H(T)$. Assume that a position in $H$ must be hedged at discrete time instants $\tau, \tau+1, \ldots, T-1$, called rebalancing instants.

Let $V$ be a self-financing portfolio composed of these two assets. The value of the portfolio at time $\tau$ is $V(\tau)$, with $V(0)$ being the initial wealth. An optimal hedging strategy, $\{u(\tau)\}_{\tau=0, \cdots, T-1}$ (optimal control law), can be obtained by solving the mean-variance hedging problem, which gives the best approximation by means of self-financing trading strategies, with the optimality criterion being the expected squared replication error.

Defining $E_{\tau}^{P}[\cdot]$ as the conditional expectation operator w.r.t. probability measure $P$ given the filtration $\mathcal{F}_{\tau}$, the value function to be minimized at time $0, \tilde{\mathcal{J}}_{T}$, is given by:

$$
\tilde{\mathcal{J}}_{T}(0)=\min _{V(0), u_{0}, \ldots, u_{T-1}} E_{0}^{P}\left[(V(T)-H(T))^{2}\right],
$$

with $V(0)$ being $\mathcal{F}_{0}$-measurable, and $u_{\tau} \mathcal{F}_{\tau}$-measurable, $\tau=0,1, \cdots, T-1$.

Let $\Delta X(\cdot)$, the discounted gain process of $S$, be given by:

$$
\Delta X(\tau+1)=\frac{S(\tau+1)}{S^{0}(\tau+1)}+\frac{\delta(\tau+1)}{S^{0}(\tau+1)}-\frac{S(\tau)}{S^{0}(\tau)},
$$

with $\delta(\tau)$ corresponding to the dividends paid for holding the risky asset $S$ between discrete-time instants $\tau$ and $\tau+1$. 
The value $V(\tau), \mathcal{F}_{\tau}$-measurable, evolves according to the optimal control law, i.e. it is the portfolio generated by the control policy $\{u(\tau)\}_{\tau=0, \cdots, T-1}$. At time $\tau=0$, the value of this portfolio is $V(0)$. It can be shown that:

$$
V(\tau+1)=e^{r \Delta t} V(\tau)+S^{0}(\tau+1) u(\tau) \Delta X(\tau+1) .
$$

Under these conditions, the solution of the optimisation problem defined in (2.3) is, as shown in Černý [1], given by

$$
\begin{gathered}
\tilde{u}(\tau)=-\frac{E_{\tau}^{P}\left\{k(\tau+1) \Delta X(\tau+1)\left(\frac{V(\tau)}{S^{0}(\tau)}-\frac{H(\tau+1)}{S^{0}(\tau+1)}\right)\right\}}{E_{\tau}^{P}\left\{k(\tau+1)(\Delta X(\tau+1))^{2}\right\}}, \tau=0, \cdots, T-1, \\
V(0)=H(0),
\end{gathered}
$$

where:

$$
\begin{aligned}
H(\tau) & =S^{0}(\tau) E_{\tau}^{P}\left\{m_{T, \tau}^{P \rightarrow Q} \frac{H(T)}{S^{0}(T)}\right\}, \\
m_{T, \tau}^{P \rightarrow Q} & =\prod_{j=\tau}^{T-1} m_{j+1, j}^{P \rightarrow Q}, \\
m_{j+1, j}^{P \rightarrow Q} & =\frac{k(j+1)-\frac{E_{j}^{P}\{k(j+1) \Delta X(j+1)\}}{E_{j}^{P}\left\{k(j+1)(\Delta X(j+1))^{2}\right\}} k(j+1) \Delta X(j+1)}{E_{j}^{P}\{k(j+1)\}-\frac{\left(E_{j}^{P}\{k(j+1) \Delta X(j+1)\}\right)^{2}}{E_{j}^{P}\left\{k(j+1)(\Delta X(j+1))^{2}\right\}}}, \\
\frac{k(\tau)}{R_{f}^{2}(\tau)} & =E_{\tau}^{P}\{k(\tau+1)\}-\frac{\left(E_{\tau}^{P}\{k(\tau+1) \Delta X(\tau+1)\}\right)^{2}}{E_{\tau}^{P}\left\{k(\tau+1)(\Delta X(\tau+1))^{2}\right\}}, \\
k(T) & =1 .
\end{aligned}
$$

Extending the work of Černý [1] to the case where the price of a risky asset price is represented by a lognormal geometric brownian motion with constant parameters, as in (2.2), we obtain explicit expressions for both the mean-value process, $H(\tau)$, of the option to be hedged, and the optimal control, $\tilde{u}(\tau)$, to be applied at the rebalancing instant $\tau$. The main results are given by Theorems 2.1 and 2.2 stated below. Full proofs can be found in Maiali [6].

In what follows we use the following notation:

1. $E_{l, \tau}^{Q}\{\cdot\}$ is the conditional expectation operator, as defined before. The subscript $l$ is used just to explicitly show the dependence of the operator on $l$, which will be introduced due to the change from the probability measure $P$ to $Q$, with $Q$ being a probability measure whose Radon-Nikodým derivative with respect to $P$ will depend on $l$. The same holds for $E_{l, \tau}^{S}\{\cdot\}$ and $E_{l, \tau}^{T}\{\cdot\}$.

2. $\mathbb{I}_{A}(x)$ represents the indicator function of $x$ w.r.t. the set $A$.

3. $C_{p, l}$ is the $l$-th element of the set $C_{p}, 1 \leq l \leq\left(\begin{array}{l}n \\ p\end{array}\right)$, whose elements are subsets formed by $p$ elements, $0 \leq p \leq n$, taken from the set $\{1, \cdots, n\}$. 
4. $\sigma_{l}(\tau+j-1)=\sigma \mathbb{I}_{C_{p, l}}(j)$.

Theorem 2.1 Let $H(\tau)$ and $m_{T, \tau}^{P \rightarrow Q}$ be given by (2.8) and (2.9), respectively. Then, $H(\tau)$ can be written as:

$$
H(\tau)=e^{-r(T-\tau) \Delta t} \sum_{p=0}^{n}\left(a_{0}^{n-p} a_{1}^{p} \sum_{l=1}^{\left(\begin{array}{l}
n \\
p
\end{array}\right)} E_{l, \tau}^{Q}\{H(T)\}\right),
$$

where:

$$
a_{0}=\frac{e^{(r-\mu) \Delta t}-e^{\sigma^{2} \Delta t}}{1-e^{\sigma^{2} \Delta t}}, \quad a_{1}=1-a_{0}=\frac{1-e^{(r-\mu) \Delta t}}{1-e^{\sigma^{2} \Delta t}},
$$

with $Q$ being a probability measure whose Radon-Nikodým derivative is given by:

$$
\begin{aligned}
& \frac{d Q}{d P}=\exp \left(\sum_{j=1}^{n}\left(\sigma \mathbb{I}_{C_{p, l}}(j) \Delta W^{P}(\tau+j)-\frac{1}{2}\left(\sigma \mathbb{I}_{C_{p, l}}(j)\right)^{2} \Delta t\right)\right) \\
& =\exp \left(\sum_{j=1}^{T-\tau}\left(\sigma_{l}(\tau+j-1) \Delta W^{P}(\tau+j)-\frac{1}{2} \sigma_{l}^{2}(\tau+j-1) \Delta t\right)\right) .
\end{aligned}
$$

Theorem 2.2 Let $\Delta X(\tau+1), V(\tau), k(\tau+1)$, and $H(\tau+1)$ be given respectively by (2.4), (2.5), (2.11), and (2.13). Then, the optimal control $\tilde{u}(\tau)$, given by (2.6), can be written as:

$$
\begin{aligned}
\tilde{u}(\tau) & =\frac{e^{-r(T-\tau) \Delta t} \sum_{p=0}^{n} a_{0}^{n-p} a_{1}^{p} \sum_{l=1}^{\left(\begin{array}{l}
n \\
p
\end{array}\right)}\left(e^{(\mu-r) \Delta t} E_{l, \tau}^{S}\{H(T)\}-E_{l, \tau}^{T}\{H(T)\}\right)}{S(\tau)\left(e^{\left(2 \mu-2 r+\sigma^{2}\right) \Delta t}-2 e^{(\mu-r) \Delta t}+1\right)} \\
& -\frac{V(\tau)\left(e^{(\mu-r) \Delta t}-1\right)}{S(\tau)\left(e^{\left(2 \mu-2 r+\sigma^{2}\right) \Delta t}-2 e^{(\mu-r) \Delta t}+1\right)},
\end{aligned}
$$

where $S$ and $T$ are probability measures whose Radon-Nikodýn derivatives are given by:

$$
\begin{gathered}
\frac{d S}{d P}=\exp \left(\sum_{j=1}^{n}\left(\Lambda_{l}(\tau+j-1) \Delta W^{P}(\tau+j)-\frac{1}{2} \Lambda_{l}^{2}(\tau+j-1) \Delta t\right)\right), \\
\Lambda_{l}(\tau+j-1)= \begin{cases}\sigma_{l}(\tau+j-1) & j=2, \cdots, T-\tau \\
\sigma & j=1,\end{cases}
\end{gathered}
$$




$$
\begin{gathered}
\frac{d T}{d P}=\exp \left(\sum_{j=1}^{n}\left(\Gamma_{l}(\tau+j-1) \Delta W^{P}(\tau+j)-\frac{1}{2} \Gamma_{l}^{2}(\tau+j-1) \Delta t\right)\right) \\
\Gamma_{l}(\tau+j-1)= \begin{cases}\sigma_{l}(\tau+j-1) & j=2, \cdots, T-\tau \\
0 & j=1 .\end{cases}
\end{gathered}
$$

\section{Application: European call options}

Here we apply the results obtained in the previous section to the case in which the derivative to be hedged is a European vanilla call option. We derive closedform solutions for both the mean-value process, $H(\tau)$, of the option to be hedged, and the optimal control, $\tilde{u}(\tau)$, to be applied at rebalancing instant $\tau$. It should be noted that their final expressions are extensions of the B\&S formulae. These closed-form solutions eliminate the recursiveness of previously proposed models, thus producing considerable computational gains. Similar procedures would lead to closed-form solutions for the case of European vanilla put options.

Numerical analyses are presented in Section 4. As in the previous section, the main results are presented in the form of theorems, with their full proofs being found in Maiali [6].

Theorem 3.1 Consider an European vanilla call option whose payoff is given by $H(T)=(S(T)-K)^{+}$. Equations (2.13) and (2.16) can be written as:

$$
\begin{aligned}
H(\tau) & =\sum_{p=0}^{n}\left(( \begin{array} { l } 
{ n } \\
{ p }
\end{array} ) a _ { 0 } ^ { n - p } a _ { 1 } ^ { p } \left[e^{\left[(\mu-r-\rho)(T-\tau)+\sigma^{2} p\right] \Delta t} S(\tau) N\left(d_{R}\right)\right.\right. \\
& \left.\left.-e^{-r(T-\tau) \Delta t} K N\left(d_{Q}\right)\right]\right)
\end{aligned}
$$

where:

$$
\begin{aligned}
& d_{Q}=\frac{\ln \left(\frac{S(\tau)}{K}\right)+\left(\mu-\rho-\frac{1}{2} \sigma^{2}\right)(T-\tau) \Delta t+\sigma^{2} p \Delta t}{\sigma \sqrt{(T-\tau) \Delta t}}, \\
& d_{R}=d_{Q}+\sigma \sqrt{(T-\tau) \Delta t}
\end{aligned}
$$

and

$$
\tilde{u}(\tau)=\frac{e^{-r(T-\tau) \Delta t} \sum_{p=0}^{n} a_{0}^{n-p} a_{1}^{p} \sum_{l=1}^{\left(\begin{array}{l}
n \\
p
\end{array}\right)}\left(e^{(\mu-r) \Delta t} E_{l, \tau}^{S}\{H(T)\}-E_{l, \tau}^{T}\{H(T)\}\right)}{S(\tau)\left(e^{\left(2 \mu-2 r+\sigma^{2}\right) \Delta t}-2 e^{(\mu-r) \Delta t}+1\right)}
$$




$$
-\frac{V(\tau)\left(e^{(\mu-r) \Delta t}-1\right)}{S(\tau)\left(e^{\left(2 \mu-2 r+\sigma^{2}\right) \Delta t}-2 e^{(\mu-r) \Delta t}+1\right)},
$$

where:

$$
\begin{aligned}
E_{l, \tau}^{S}\{H(T)\} & =S(\tau) e^{(\mu-\rho)(T-\tau) \Delta t+\sigma^{2} \Delta t\left(\varphi_{p, l}+1\right)} N\left(d_{U}\right)-K N\left(d_{S}\right), \\
E_{l, \tau}^{T}\{H(T)\} & =S(\tau) e^{(\mu-\rho)(T-\tau) \Delta t+\sigma^{2} \Delta t \varphi_{p, l} N\left(d_{V}\right)-K N\left(d_{T}\right),} \\
d_{S} & =\frac{\ln \left(\frac{S(\tau)}{K}\right)+\left(\mu-\rho-\frac{1}{2} \sigma^{2}\right)(T-\tau) \Delta t+\sigma^{2} \Delta t\left(\varphi_{p, l}+1\right)}{\sigma \sqrt{(T-\tau) \Delta t}}, \\
d_{U} & =d_{S}+\sigma \sqrt{(T-\tau) \Delta t}, \\
d_{T} & =\frac{\ln \left(\frac{S(\tau)}{K}\right)+\left(\mu-\rho-\frac{1}{2} \sigma^{2}\right)(T-\tau) \Delta t+\sigma^{2} \Delta t \varphi_{p, l}}{\sigma \sqrt{(T-\tau) \Delta t}} \\
d_{V} & =d_{T}+\sigma \sqrt{(T-\tau) \Delta t}, \\
\varphi_{p, l} & = \begin{cases}p-1 & \text { if } p \neq 0 ; 1 \leq l \leq\left(\begin{array}{l}
n-1 \\
p-1
\end{array}\right) \\
p & \text { if } p \neq 0 ;\left(\begin{array}{l}
n-1 \\
p-1
\end{array}\right)<l \leq\left(\begin{array}{l}
n \\
p
\end{array}\right) .\end{cases}
\end{aligned}
$$

\section{Numerical results}

Here the results obtained in Section 3 are applied to European call options maturing in 6 and 12 months. Consider that $r=17 \%$ per annum (present level of Brazilian interest rates), that the current value of the underlying asset is $S=100$, and that it pays no dividend $(\rho=0)$.

Results for three different strikes are compared, $K=95, K=100$, and $K=115$, corresponding to in-the-money, at-the-money and out-of-the-money options, respectively. For each possible situation (maturity date and strike) we observe the effects of different expected rates of return, $\mu$, with $\mu=10 \%$ and $\mu=20 \%$, different volatilities, $\sigma$, with $\sigma=20 \%$ and $\sigma=40 \%$, and different number of rebalancing instants, $n$, with $n=6$ and $n=10$. Paths of the underlying asset are simulated according to (2.1). For each path there is a payoff, $H(T)$, which is compared with the value of the hedging porfolio at maturity, $V(T)$. The hedging error, expressed as the present value of the square root of the meansquared difference between the option's payoff and hedging portfolio at maturity, is calculated relative to the option's current value. The procedure is repeated for two hedging methods: (i) the dynamic programming approach (DP) proposed in Section 3; and (ii) the B\&S approach (delta-hedging). Results for the error incurred by both methods, as well as the relative error of DP with respect to B\&S, are presented for each combination of parameters. Results for in-, at- and out-of-themoney call options maturing in 6 and 12 months are given in Tables 1, 2 and 3, respectively. Hedging errors for both methods (columns "error DP" and "error 
Table 1: $K=95$ (in-the-money); $r=17 \%, S=100$.

\begin{tabular}{lll|lll|lll}
\hline \hline & & & \multicolumn{3}{|c}{$\mathrm{T}=6$ months } & \multicolumn{3}{c}{$\mathrm{T}=12$ months } \\
\hline $\mathrm{n}$ & $\mu$ & $\sigma$ & error & error & rel. & error & error & $\begin{array}{l}\text { rel. } \\
\text { error }\end{array}$ \\
\hline \multirow{3}{*}{6} & $10 \%$ & $20 \%$ & $13.07 \%$ & $12.93 \%$ & $-1.11 \%$ & $10.33 \%$ & $10.20 \%$ & $-1.24 \%$ \\
& $10 \%$ & $40 \%$ & $35.94 \%$ & $35.50 \%$ & $-1.21 \%$ & $28.24 \%$ & $27.59 \%$ & $-2.31 \%$ \\
& $20 \%$ & $20 \%$ & $8.88 \%$ & $8.84 \%$ & $-0.41 \%$ & $6.01 \%$ & $5.95 \%$ & $-1.09 \%$ \\
& $20 \%$ & $40 \%$ & $32.18 \%$ & $31.95 \%$ & $-0.71 \%$ & $25.24 \%$ & $24.88 \%$ & $-1.43 \%$ \\
\hline \multirow{3}{*}{10} & $10 \%$ & $20 \%$ & $12.49 \%$ & $12.17 \%$ & $-2.50 \%$ & $10.30 \%$ & $9.86 \%$ & $-4.32 \%$ \\
& $10 \%$ & $40 \%$ & $37.90 \%$ & $37.40 \%$ & $-1.33 \%$ & $31.77 \%$ & $31.04 \%$ & $-2.32 \%$ \\
& $20 \%$ & $20 \%$ & $8.30 \%$ & $8.29 \%$ & $-0.15 \%$ & $5.70 \%$ & $5.67 \%$ & $-0.45 \%$ \\
& $20 \%$ & $40 \%$ & $33.28 \%$ & $33.19 \%$ & $-0.26 \%$ & $27.23 \%$ & $27.07 \%$ & $-0.60 \%$ \\
\hline \hline
\end{tabular}

Table 2: $K=100$ (at-the-money); $r=17 \%, S=100$.

\begin{tabular}{lll|lll|lll}
\hline \hline & & & \multicolumn{3}{|c}{$\mathrm{T}=6$ months } & \multicolumn{3}{c}{$\mathrm{T}=12$ months } \\
\hline $\mathrm{n}$ & $\mu$ & $\sigma$ & error & error & rel. & error & error & $\begin{array}{l}\text { rel. } \\
\text { error }\end{array}$ \\
\hline \multirow{3}{*}{6} & $10 \%$ & $20 \%$ & $30.31 \%$ & $30.06 \%$ & $-0.84 \%$ & $17.57 \%$ & $17.37 \%$ & $-1.12 \%$ \\
& $10 \%$ & $40 \%$ & $48.33 \%$ & $47.79 \%$ & $-1.11 \%$ & $34.31 \%$ & $33.57 \%$ & $-2.15 \%$ \\
& $20 \%$ & $20 \%$ & $21.71 \%$ & $21.67 \%$ & $-0.19 \%$ & $11.64 \%$ & $11.55 \%$ & $-0.71 \%$ \\
& $20 \%$ & $40 \%$ & $46.53 \%$ & $46.27 \%$ & $-0.56 \%$ & $32.23 \%$ & $31.84 \%$ & $-1.20 \%$ \\
\hline \multirow{3}{*}{10} & $10 \%$ & $20 \%$ & $31.32 \%$ & $30.75 \%$ & $-1.82 \%$ & $18.95 \%$ & $18.26 \%$ & $-3.66 \%$ \\
& $10 \%$ & $40 \%$ & $52.45 \%$ & $51.85 \%$ & $-1.16 \%$ & $39.28 \%$ & $38.43 \%$ & $-2.19 \%$ \\
& $20 \%$ & $20 \%$ & $21.65 \%$ & $21.65 \%$ & $-0.02 \%$ & $11.57 \%$ & $11.55 \%$ & $-0.24 \%$ \\
& $20 \%$ & $40 \%$ & $49.22 \%$ & $49.10 \%$ & $-0.24 \%$ & $35.16 \%$ & $35.00 \%$ & $-0.46 \%$ \\
\hline \hline
\end{tabular}

B\&S") as well as the DP error relative to that of the B\&S approach (column "relative error") are presented.

It can be observed that, in all cases, whenever the expected rate of return, $\mu$, assumes values close to the risk-free rate $r$ (e.g. $r=17 \%$ and $\mu=20 \%$ ), the results from the $\mathrm{B} \& \mathrm{~S}$ model approach, but are consistently worse than those obtained by the DP model, as it should be expected, since in a B\&S risk-neutral setting, an Itô diffusion with rate $\mu$ corresponds to a risk-neutral diffusion with rate $r$. Conversely, whenever $\mu$ and $r$ are apart (e.g. $r=17 \%$ and $\mu=10 \%$ ), the DP model behaves considerably better, as the assumptions of the B\&S model no longer hold.

Since both models are linear approximations for $H(\cdot)$, the results indicate that, irrespective of the moneyness of the option to be hedged, for a small number of rebalancing instants (e.g. $n=6$ ), and high volatility (e.g. $\sigma=40 \%$ ), both methods 
Table 3: $K=115$ (out-of-the money); $r=17 \%, S=100$.

\begin{tabular}{lll|lll|lll}
\hline \hline & & & \multicolumn{3}{|c}{$\mathrm{T}=6$ months } & \multicolumn{3}{c}{$\mathrm{T}=12$ months } \\
\hline $\mathrm{n}$ & $\mu$ & $\sigma$ & error & error & rel. & error & error & $\begin{array}{l}\text { rel. } \\
\text { error }\end{array}$ \\
\hline \multirow{3}{*}{6} & $10 \%$ & $20 \%$ & $65.65 \%$ & $65.17 \%$ & $-0.73 \%$ & $37.95 \%$ & $37.60 \%$ & $-0.95 \%$ \\
& $10 \%$ & $40 \%$ & $67.99 \%$ & $67.40 \%$ & $-0.87 \%$ & $46.00 \%$ & $45.21 \%$ & $-1.71 \%$ \\
& $20 \%$ & $20 \%$ & $79.94 \%$ & $79.84 \%$ & $-0.13 \%$ & $33.31 \%$ & $33.24 \%$ & $-0.20 \%$ \\
& $20 \%$ & $40 \%$ & $74.37 \%$ & $74.20 \%$ & $-0.22 \%$ & $47.76 \%$ & $47.44 \%$ & $-0.66 \%$ \\
\hline \multirow{3}{*}{10} & $10 \%$ & $20 \%$ & $67.72 \%$ & $66.59 \%$ & $-1.68 \%$ & $42.17 \%$ & $40.93 \%$ & $-2.95 \%$ \\
& $10 \%$ & $40 \%$ & $70.81 \%$ & $70.07 \%$ & $-1.05 \%$ & $51.79 \%$ & $50.71 \%$ & $-2.08 \%$ \\
& $20 \%$ & $20 \%$ & $86.55 \%$ & $86.54 \%$ & $-0.01 \%$ & $36.27 \%$ & $36.27 \%$ & $-0.02 \%$ \\
& $20 \%$ & $40 \%$ & $80.19 \%$ & $80.11 \%$ & $-0.10 \%$ & $54.01 \%$ & $53.85 \%$ & $-0.31 \%$ \\
\hline \hline
\end{tabular}

produce significant hedging errors. Nevertheless, even in this situation, it can be observed that the proposed method outperforms the B\&S model. It should be noted that, as $n$ increases, although results produced by the DP model converge to those obtained by the B\&S model (following the assumption of infinitesimal rebalancing instants from the latter), the proposed method consistently incurs less hedging errors than those obtained the $\mathrm{B} \& \mathrm{~S}$ approach, apart from results for small $n$, in which case both models behave poorly.

The situation that indicates the best relative performance of the proposed method is the case of small volatilities (see results for $\sigma=20 \%$ in Tables 1, 2 and 3), as the payoff of the option becomes less unpredictable.

\section{Summary and concluding remarks}

In this work we have analysed the mean-variance hedging problem of a continuous state space financial model with the rebalancing strategies for the hedging portfolio taken at discrete times. We have derived an expression for the optimal selffinancing mean-variance hedging strategy problem, considering any given payoff in an incomplete market environment. As an application of the proposed method, we have obtained closed-form solutions for the value European vanilla call options and for the amount of the corresponding underlying asset to be bought or sold for hedging purposes (optimal control law).

The results showed that the proposed solution is consistently better than the $\mathrm{B} \& \mathrm{~S}$ delta-hedging approach for all possible combinations of parameters considered. As expected, the proposed method presents relatively better results, especially when the market structure does not follow their basic assumptions. The method is flexible enough with regard to the determination of optimal hedging strategies to be applied to a broad variety of European-style derivatives and stochastic price processes of their underlying asset. In particular, our current 
research is concentrated towards: (i) obtaining closed-forms solutions for other instruments; and (ii) modelling asset prices whose dynamics are represented by jump-diffusions and/or stochastic volatility models.

\section{Acknowledgments}

O.L.V. Costa was partially supported by CNPq (Brazilian National Research Council), grants 472920/03-0 and 304866/03-2, FAPESP (Rese-arch Council of the State of São Paulo), grant 03/06736-7, PRONEX, grant 015/98, and IMAGIMB.

\section{References}

[1] Černý, A., Dynamic programming and mean-variance hedging in discrete time. Applied Mathematical Finance, 1(11), pp. 1-25, 2004.

[2] Schäl, M., On quadratic cost criteria for option hedging. Mathematics of Operations Research, 1(19), pp. 121-131, 1994.

[3] Schweizer, M., Variance-optimal hedging in discrete time. Mathematics of Operations Research, 1(20), pp. 1-32, 1995.

[4] Bertsimas, D., Kogan, L. \& Lo, A.W., Hedging derivative securities in incomplete market: An $\epsilon$-arbitrage approach. Operations Research, 3(49), pp. 372-397, 2001.

[5] Black, F. \& Scholes, M., The pricing of options and corporate liabilities. Journal of Political Economy, (81), pp. 637-654, 1973.

[6] Maiali, A.C., Stochastic optimal control at discrete time and continuous state space applied to derivatives. Ph.D. thesis, Escola Politécnica - Universidade de São Paulo, 2006.

[7] Pham, H., Rheinländer, T. \& Schweizer, M., Mean-variance hedging for continuous processes: New results and examples. Finance and Stochastics, (2), pp. 173-198, 1998.

[8] Laurent, J.P. \& Pham, H., Dynamix programming and mean-variance hedging. Finance and Stochastics, 1(3), pp. 83-110, 1999.

[9] Schweizer, M., Mean-variance hedging for general claims. The Annals of Applied Probability, 1(2), pp. 171-179, 1992.

[10] Schweizer, M., Approximation pricing and the variance-optimal martingale measure. The Annals of Applied Probability, 1(24), pp. 206-236, 1996. 